\title{
Disruption of the Myostatin Gene in Porcine Primary Fibroblasts and Embryos Using Zinc-Finger Nucleases
}

\author{
Xian-Ju Huang ${ }^{1,3}$, Hong-Xiao Zhang ${ }^{1,3}$, Huili Wang ${ }^{2}$, Kai Xiong ${ }^{1}$, Ling Qin ${ }^{1}$, and Honglin Liu ${ }^{1, *}$
}

\begin{abstract}
Myostatin represses muscle growth by negatively regulating the number and size of muscle fibers. Myostatin lossof-function can result in the double-muscling phenotype and increased muscle mass. Thus, knockout of myostatin gene could improve the quality of meat from mammals. In the present study, zinc finger nucleases, a useful tool for generating gene knockout animals, were designed to target exon 1 of the myostatin gene. The designed ZFNs were introduced into porcine primary fibroblasts and early implantation embryos via electroporation and microinjection, respectively. Mutations around the ZFNs target site were detected in both primary fibroblasts and blastocysts. The proportion of mutant fibroblast cells and blastocyst was $4.81 \%$ and $5.31 \%$, respectively. Thus, ZFNs can be used to knockout myostatin in porcine primary fibroblasts and early implantation embryos.
\end{abstract}

\section{INTRODUCTION}

Endogenous gene knockout is a powerful tool for analyzing gene function, optimizing livestock production, and generating animal models for human genetic diseases. Recent work developed a method of gene disruption using zinc-finger nucleases (ZFNs). ZFNs can generate double-strand breaks (DSBs) at specific sequences. In mammals, DSBs are repaired primarily via the error-prone non-homologous end-joining (NHEJ) mechanism. The NHEJ repair pathway leads to gene disruption due to small insertions and deletions at the DSBs. The ZFNs technology is a useful approach for generating knockout mutants because it is not necessary for cells to undergo long-term drug selection; therefore, this method improves the proliferation potential of mammalian cells intended for somatic cell nuclear

\footnotetext{
${ }^{1}$ College of Animal Sciences and Technology, Nanjing Agricultural University, ${ }^{2}$ Institute of Animal Science, Jiangsu Academy of Agricultural Sciences, Nanjing, Jiangsu, China, ${ }^{3}$ These authors contributed equally to this work.

*Correspondence: liuhonglin @ njau.edu.cn
}

Received 17 July, 2013; revised 19 March, 2014; accepted 21 March, 2014; published online 21 April, 2014

Keywords: gene, knockout, myostatin, porcine, zinc-finger nucleases (ZFNs) transfer (SCNT). Currently, gene knockout has been accomplished with ZFNs-coding mRNA or DNA in a number of organisms including mice, rats, pigs, and cattle (Carbery et al., 2010; Hauschild et al., 2011; Li et al., 2013; Mashimo et al., 2010; Yang et al., 2011; Yu et al., 2011). The ZFNs platform may overcome the inherent limitations of genetic modification techniques in mammals that not involve embryonic stem (ES) cells.

The ZFNs platform is not the only method available for gene knockout in mammals. An alternate method of genome editing uses transcription activator-like effector nucleases (TALENs), which provide greater flexibility in selecting target sequences than that of ZFNs (Cermak et al., 2011). Clustered regulatory interspaced short palindromic repeats (CRISPR)-based systems utilize RNA-guided DNA endonucleases that function in the sequence-specific silencing of invading foreign DNA (Wiedenheft et al., 2012). All three gene knockout methods are transformative tools with the potential to revolutionize biological research.

The myostatin gene was discovered first in mice (McPherron et al., 1997), and is an attractive target for gene knockout in livestock. It regulates the number and size of muscle fibers by repressing muscle growth. Disruption of the myostatin gene has been studied in cattle (Grobet et al., 1997) and humans (Schuelke et al., 2004). Myostatin loss-of-function can result in the double-muscling phenotype and increase muscle mass. Thus, disruption of the myostatin gene in food animals could potentially produce leaner meat. In this study, we tested a pair of ZFNs that showed high activity at the first exon of the porcine myostatin gene. Our results demonstrate that ZFNs disrupt myostatin in porcine fetal fibroblasts and embryos. To the best of our knowledge, this is the first evidence of myostatin gene disruption using ZFNs in this system.

\section{MATERIALS AND METHODS}

\section{Design of ZFNs}

The ZFNs were designed to bind and cleave the first exon of the porcine myostatin gene, and were purchased from SigmaAldrich.

Cell culture and transfection of primary porcine fetal fibroblasts Primary porcine fetal fibroblasts were obtained from the 30 days-old fetus of a Meishan (China) pig. Skin biopsies isolated from the body of the fetus were used to prepare porcine prima- 
ry fibroblasts. A total of $10^{6}$ cells were trypsinized $(0.25 \%)(\mathrm{w} / \mathrm{v})$, washed with phosphate-buffered saline (PBS) (gibco), to centrifugate (1200 r/min, $4 \mathrm{~min}$ ) and suspended in $100 \mu \mathrm{l}$ Nucleofector Solution (Lonza) containing $2 \mu \mathrm{g}$ DNA of ZFNs or pEGFP. $\mathrm{N} 1$ plasmids. Cells were then transfected with the plasmids using program T-016 (Lonza). Treated cells were placed in a T25 flask (Nunc) and cultured at $37^{\circ} \mathrm{C}, 5 \% \mathrm{CO}_{2}$ for 2 days.

Production of parthenogenetically activated embryos Porcine ovaries were collected from a local abattoir and stored in sterile PBS containing $100 \mathrm{IU} / \mathrm{ml}$ penicillin and $0.05 \mathrm{mg} / \mathrm{ml}$ streptomycin at $30-35^{\circ} \mathrm{C}$ during transportation. Cumulus oocyte complexes (COCs) with intact cumulus cell layers and uniform cytoplasm were selected for maturation in vitro. Selected COCs were washed three times with PZM-3 medium, transferred to pre-equilibrated PZM-3 medium and then incubated for $44-48 \mathrm{~h}$ at $38.5^{\circ} \mathrm{C}, 5 \% \mathrm{CO}_{2}$. COCs were transferred to $0.1 \%(\mathrm{w} / \mathrm{v})$ hyaluronidase in Dulbecco's PBS (DPBS) (gibco) to remove cumulus cells. Oocytes with clear, intact cell membranes, perivitelline space, and a first polar body were selected for parthenogenetic activation (PA) and transferred to activation medium (2\%FBS /TCM199)(Life Technologies). Activation was performed via direct current (DC) $1.2 \mathrm{kV} / \mathrm{cm}$ for $30 \mu \mathrm{s}$. Subsequently, oocytes were transferred into $5 \mu \mathrm{g} / \mathrm{ml}$ Cytochalasin B (CB) and incubated for $3 \mathrm{~h}$. Oocytes were then washed three times and cultured in fresh $\mathrm{PZM}-3$ medium at $38.5^{\circ} \mathrm{C}, 5 \% \mathrm{CO}_{2}$.

Microinjection of ZFNs-coding mRNA into porcine embryos

ZFNs-coding mRNA was transcribed and polyadenylated from linear ZFNs-coding DNA using the mMESSAGE mMACHINE ${ }^{\circledR}$ and $\operatorname{Poly}(\mathrm{A})$ Tailing kits (Ambion), then purified using the MEGAclear $^{\text {TM }}$ kit (Ambion). Two ZFNs monomer-coding mRNAs were mixed at a final concentration of $20 \mathrm{ng} / \mu \mathrm{l}$, and approximately $10 \mathrm{pl}$ was microinjected into embryos cytoplasm at 8-10 $\mathrm{h}$ after PA. Using a microscope (IX71, Olympus), manipulators (Olympus) and pressure instrument (Narishige) for microjection and was performed on the PA embryos. After microinjection, embryos were cultured in PZM3 medium at $38.5^{\circ} \mathrm{C}, 5 \% \mathrm{CO}_{2}$ until develop to blastocyst.

\section{Immunofluorescence}

Cells were washed in PBS containing $1 \mathrm{mg} / \mathrm{ml}$ bovine serum albumin (BSA), and fixed in 3.7\% paraformaldehyde (PFA) for $40 \mathrm{~min}$ at room temperature (RT) and then were permeabilised with $0.5 \%$ Triton X- 100 for 30 min at RT. After washing, cells were further incubated at $4^{\circ} \mathrm{C}$ over night with antibody that recognized FLAG (5 $\mu \mathrm{g} / \mathrm{ml}$, Sigma Aldrich). Secondly, AlexaFluor 488- conjugated antibody (Life Technology) was diluted 1: 1000 in which the cells were incubated for $1 \mathrm{~h}$ in the dark at RT. After washed three times, cells were mounted in $3 \mathrm{mg} / \mathrm{ml} \mathrm{4,6-} \mathrm{diami-}$ dino-2-phenylindole (DAPI; Sigma aldrich) for $25 \mathrm{~min}$ at RT. And then, cells were moved into $20 \mu \mathrm{l} \%$ Vectashield antibleaching solution (Vector Laboratories). Fluorescence was captured by Carl Zeiss LSM 700 and saved in TIFF format. Besides, cells were transfected with pEGFP-N1 were not fixed and permeabilised but directed mounted in $10 \mu \mathrm{g} / \mathrm{ml}$ Hoechst 33342 (Sigma) for $15 \mathrm{~min}$ at room temperature. Then, fluorescence was detected at $10 \times 40$ using an inverted microscope (IX71, Olympus).

T7 endonuclease I mutation-detection assay

ZFNs-induced fibroblast and oocyte mutations were detected using a T7E1 assay (New England BioLabs, USA) following the manufacturer's protocol and a recently reported method (Kim et al., 2009). ZFNs-treated porcine genomic DNA was extracted from the following two samples: (1) fibroblasts that were transfect 2 days later, and (2) embryos that had developed to the blastula stage. PCR analysis of fibroblast genomic DNA was performed using the myostatin primers MSTN-1-F (5'AAAGGAAGAAATAAGAACAAGGA-3') and MSTN-1-R (5'TTACACTCTGTAGGCATGGTAAT-3') under the following conditions: $95^{\circ} \mathrm{C}$ for $4 \mathrm{~min} ; 36$ cycles of $94^{\circ} \mathrm{C}$ for $30 \mathrm{~s}, 58^{\circ} \mathrm{C}$ for $30 \mathrm{~s}, 72^{\circ} \mathrm{C}$ for $40 \mathrm{~s}$; and a final extension at $72^{\circ} \mathrm{C}$ for $5 \mathrm{~min}$. PCR analysis of embryonic DNA was performed in two steps. Step 1 employed myostatin primers MSTN-P1-F (5'-GTGGAG CAAGAGCCAATCATAGA-3') and MSTN-P1-R (5'-CAGCAGC TTTCAGTCTCATTAGTTTAT-3') under the following conditions: $95^{\circ} \mathrm{C}$ for $4 \mathrm{~min} ; 36$ cycles of $94^{\circ} \mathrm{C}$ for $30 \mathrm{~s}, 58^{\circ} \mathrm{C}$ for $30 \mathrm{~s}$, $72^{\circ} \mathrm{C}$ for $50 \mathrm{~s}$; and a final extension at $72^{\circ} \mathrm{C}$ for $7 \mathrm{~min}$. In Step 2 , the PCR amplicons from Step 1 were purified, then a nested PCR was performed using primers MSTN-1-F and MSTN-1-R, using the same cycling conditions described for amplification of fibroblast genomic DNA. The denatured and annealed DNA fragments were treated with 5 units T7E 1 at $37^{\circ} \mathrm{C}$ for $30 \mathrm{~min}$, and then analyzed using agarose gel electrophoresis.

DNA sequence analysis of myostatin in cells and embryos Based on the results of the T7E1 assay, candidate mutant DNA fragments from ZFNs-treated fibroblasts and embryos were cloned into the pMD18-T vector (TAKARA, China) and subjected to DNA-sequencing analysis. The proportion of mutants was calculated by dividing the number of mutant clones by the number of total clones analyzed.

\section{RESULTS}

ZFNs-induced myostatin mutations in porcine primary fibroblasts

We used ZFNs that designed to bind and cleave the first exon of the porcine myostatin gene, the sequence and target of which are shown in Fig. 1A. To determine the transfection efficiency, we introduced pEGFP-N1 into porcine primary fibroblasts as a positive control. $75 \%$ of primary fibroblasts cells displayed EGFP green fluorescence $24 \mathrm{~h}$ after transfection (Supplementary Fig. 1). The ZFNs had FLAG tags. Therefore, the porcine primary fibroblasts expressing ZFNs can be detected using Immunofluorescence (Fig. 1B)

The T7E1 analysis of porcine primary fibroblasts transfected with ZFNs are presented in Figs. 1C and 1D. The 325-bp bands indicate that heteroduplexes were generated in the ZFNs-treated, fibroblast-derived amplicons, suggesting that deletions occurred due to post-treatment cleavage of the ZFNs target sites (Fig. 1D). These results suggest that ZFNs can mediate myostatin mutations in porcine primary fibroblasts.

ZFNs-induced myostatin mutations were detected in parthenogenetically activated embryos

ZFNs-coding mRNA was microinjected into porcine embryos cytoplasm, and the embryos were cultured in PZM3 medium until the blastocyst stage. Genomic DNA was extracted from injected and uninjected blastocysts. Less genomic DNA was extracted from individual embryos than from fibroblasts; therefore, we used nested primers to amplify the sequence encompassing the myostatin ZFNs target site. Subsequently, a mutation-detection assay was performed on the amplicons (Figs. $2 \mathrm{~A}$ and $2 \mathrm{~B}$ ). The results showed that myostatin fragments of different lengths were present in the amplicons. In addition, we monitored the 
A TTTACCTGTTTATGCTGAT- TTGCTGGTCCCGTGGATCTG AAATGGACAAATACGACT/ CAACGACCAGGGCACCTAGAC
B

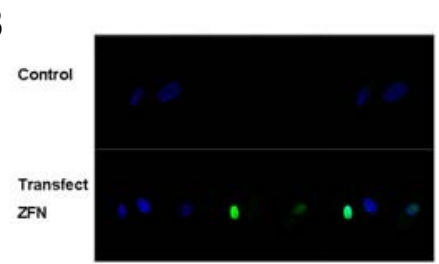

D

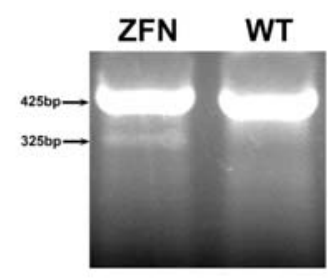

C

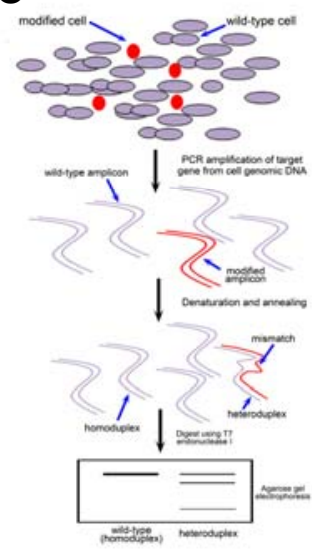

Fig. 1. ZFN-mediated disruption of myostatin gene in primary pig fibroblasts. (A) Myostatin-targeted ZFNs used in this research. The ZFNs target sequences are indicated by the red color, each of ZFN monomer recognizes 15 bases, and five bases space between the two ZFN monomers, each zincfinger protein recognizes three bases, the Fok I nucleases are acted as dimer to non-specific cleavage, identify, insertion and deletion. (B) Immunofluoresence staining analysis the myostatin-ZFN location. The DAPI staining nucleus (blue) and the Flag location myostain-ZFN (green). (C) Schematic overview of mismatch detection by T7 endonuclease I (T7E1). Genomic DNA was extracted from primary pig fibroblasts treated with ZFNs after $48 \mathrm{~h}$ of transfection, the first exon of myostatin gene that encompassing the site of ZFNs recognition was amplified by PCR, and the DNA amplicons were denaturized and annealed to form homoduplexes and heteroduplexes. The T7E1 recognizes and cleaves the mismatch DNA of heteroduplexes instead of homoduplexes, proceeding the DNA fragments analysis by agarose gel electrophoresis and the ideal result is shown. (D) T7E1 assay of ZFN cleavage in primary porcine fibroblasts. A band cleave by the T7 endonuclease I was detected at an expected size ( $\sim 325 \mathrm{bp})$, indicating the presence of heteroduplexes. ZFN, amplicons from ZFNs treated primary porcine fibroblasts; WT, amplicons from wild-type primary pig fibroblast.

Fig. 2. ZFN-mediated disruption of myostatin gene in porcine PA embryos. (A) T7E1 assay of ZFN cleavage in porcine PA embryos. The 425 bp PCR amplicon of myostatin gene is cleaved into $\sim 325$ bp and $\sim 100$ bp fragments. ZFN, the embryo mutant after ZFN coding mRNA treated; Con, the embryo treated with nonsense mRNA. (B) DNA sequencing diagram of an embryo mutant. The diagram of sequence reveals a double curve around ZFNs binding site. Arrow indicated mutant point. (C) Effect on development of PA embryos after microinjected with ZFN-coding mRNA in 1-cell. After injected with ZFNcoding mRNA in 1-cell, the developmental rate of embryos from 2-cell to blastula was shown. There was no significant difference between control and ZFN-treated group $(p>0.05)$. development of PA embryos after microinjection with ZFNs mRNA or with scramble mRNA. No significant difference was detected in the development of embryos injected with ZFNs or scramble mRNA (Fig. 2C). Myostatin expression is not normally detectable until the 21st day during porcine embryonic development (Ji et al., 1998). However, we detected ZFNs were active in the PA embryos at the blastocyst stage (7.5 days).

DNA-sequence analysis of mutants

DNA-sequence mutations were detected in 22 colonies derived from ZFNs-treated cells and 13 of 245 ZFNs-treated embryos (Fig. 3A). The efficiency of myostatin gene disruption by ZFNs was $4.81 \%$ and $5.31 \%$ in porcine primary fibroblasts and embryos, respectively. Mutations were induced in myostatin exon 1 in both primary fibroblasts and embryos, suggesting that the efficiency of ZFNs-induced mutation was not significantly different in fibroblasts and embryos. We calculated the percentage of cells harboring mutations in each mutant embryo (Fig. 3B). The proportion of mutant cells reached $100 \%$ in two embryos. In some mutant embryos, two to four different mutations were detected. 
A Cell

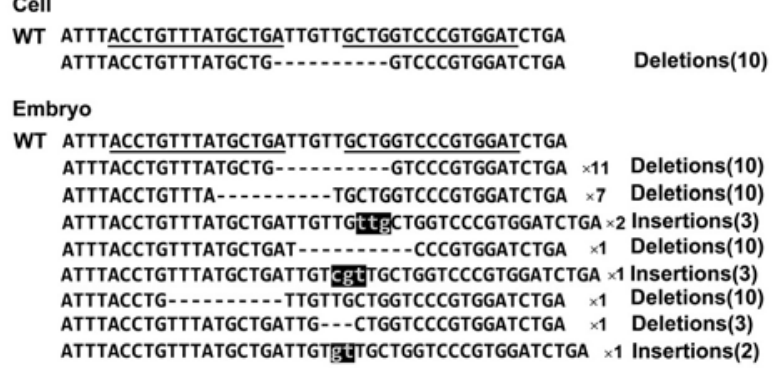

B

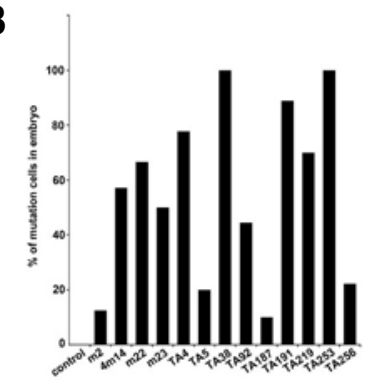

Fig. 3. Analysis of mutagenic cells and embryos. (A) DNA sequence analysis of myostatin at ZFNs target sites in primary pig fibroblasts and porcine embryos. Cloned the DNA amplicons of mutant to pMD18-T vector, single colony was isolated for DNA sequence analysis. All of the mutation type in cell and embryos are shown. The number of reads for each allele is noted to the right of the sequence. Underline, ZFNs binding sequence; dashes, deletions; small letters and black background, insertions; parenthesis, the quantity of bases insertion or deletion. The sequences only showed the mutation types. (B) The percentage of mutagenic cells in one mutagenic embryo. Ten clones were isolated each mutagenic embryos for DNA analysis, the lowest percentage of mutagenic cells in one embryo is $12.5 \%$, the highest is $100 \%$, $55 \%$ on average, indicating the high mutation rate in one mutagenic embryo.

\section{Off-target analysis}

Seven pairs of primers were provided by the manufacturer (Sigma-Aldrich) to investigate off-target activity. These primer pairs were produced by scanning the porcine genome for 5-6 bp sites that were mismatched to the ZFNs target sequence. These primer sequences are listed in Supplementary Table 1. The PCR amplification products from mutant porcine blastocyst embryos are shown in Supplementary Fig. 2A. The T7E1 cleavage assay did not detect myostatin mutations in the PCR products (Supplementary Fig. 2B). The partial silico-PCRsequencing results detected no off-target effects (Supplementary Fig. 2C). These results indicate that the ZFNs did not display off-target mutagenic activity.

\section{DISCUSSION}

This study demonstrated the feasibility of ZFNs-mediated myostatin disruption in porcine primary fibroblasts and embryos. To the best of our knowledge, this is the first time that ZFNs have been used to induce porcine myostatin mutations. Previous studies used homologous recombination (HR)-based genomic modification technologies, which require several selection markers to screen for cells with desired genetic modifications (Jin et al., 2003; Rogers et al., 2008). Therefore, HR methods result in the introduction of selective markers in the genomes of treated animals. In the current study, we applied ZFNs to generate porcine myostatin mutations. This technology does not require the transformation of antibiotic selection markers or the use of deleterious drugs to select cells with desired genetic modifications. The ZFNs approach may achieve gene knockout in less time than required for other methods.

Although it is possible to detect mutations via DNA sequencing (Yang et al., 2011), it is not an efficient method for screening all mutants. Therefore, we employed nested PCR to improve myostatin amplification from individual embryos, and then performed the T7E1 assay using the amplicons. The advantages of this approach became evident during the course of the study. In one of the embryos, DNA sequencing did not detect mutations (i.e., no double peaks in the DNA sequence near the target region). However, this embryo was identified as a mutant using the T7E1 assay (data not shown). This suggests that the T7E1 assay is more sensitive for detecting gene mutations in embryos.
We analyzed DNA sequences from ZFNs-treated fibroblast cells and embryos (Fig. 3A). Deletions and insertions were detected in ZFNs-treated cells and embryos, although we did not observe complex indels that have been reported previously (Kim et al., 2009). The average percentage of mutant cells in mutant embryos was $55 \%$. However, two mutant embryos were identified that were composed of $100 \%$ mutant cells. These data indicate that ZFNs-mediated mutations can occur during early embryonic development.

In conclusion, we demonstrate that ZFNs can efficiently disrupt myostatin in porcine primary fibroblasts and PA embryos. This technology is less time-consuming and injurious to cells than alternative approaches, and it minimizes the risks of introducing exogenous DNA fragments into the host genome. Our results may provide a future platform for improving meat production. Specifically, mutant cell lines can be generated using ZFNs, to provide SCNT donor cells for produce myostatinknockout pigs.

Note: Supplementary information is available on the Molecules and Cells website (www.molcells.org).

\section{ACKNOWLEDGMENTS}

Thanks for Prof. Dengke Pan provided ZFNs. Thanks for the help of revising language by Weikang Chen. This work was supported by the major projects of cultivate new varieties of genetically modified organisms 2008ZX08600-003 from Chinese Ministry of Agriculture.

\section{REFERENCES}

Carbery, I.D., Ji, D., Harrington, A., Brown, V., Weinstein, E.J., Liaw, L., and Cui, X. (2010). Targeted genome modification in mice using zinc-finger nucleases. Genetics 186, 451-459.

Cermak, T., Doyle, E.L., Christian, M., Wang, L., Zhang, Y., Schmidt, C., Baller, J.A., Somia, N.V., Bogdanove, A.J., and Voytas, D.F. (2011). Efficient design and assembly of custom TALEN and other TAL effector-based constructs for DNA targeting. Nucleic Acids Res. 39, e82.

Grobet, L., Martin, L.J., Poncelet, D., Pirottin, D., Brouwers, B., Riquet, J., Schoeberlein, A., Dunner, S., Menissier, F., Massabanda, J., et al. (1997). A deletion in the bovine myostatin gene causes the double-muscled phenotype in cattle. Nat. Genet. 17, 71-74.

Hauschild, J., Petersen, B., Santiago, Y., Queisser, A.L., Carnwath, 
J.W., Lucas-Hahn, A., Zhang, L., Meng, X., Gregory, P.D., Schwinzer, R., et al. (2011). Efficient generation of a biallelic knockout in pigs using zinc-finger nucleases. Proc. Natl. Acad. Sci. USA 108, $12013-12017$.

Ji, S., Losinski, R.L., Cornelius, S.G., Frank, G.R., Willis, G.M., Gerrard, D.E., Depreux, F.F., and Spurlock, M.E. (1998). Myostatin expression in porcine tissues: tissue specificity and developmental and postnatal regulation. Am. J. Physiol. 275, R12651273.

Jin, D.I., Lee, S.H., Choi, J.H., Lee, J.S., Lee, J.E., Park, K.W., and Seo, J.S. (2003). Targeting efficiency of a-1,3-galactosyl transferase gene in pig fetal fibroblast cells. Exp. Mol. Med. 35, 572577.

Kim, H.J., Lee, H.J., Kim, H., Cho, S.W., and Kim, J.S. (2009) Targeted genome editing in human cells with zinc finger nucleases constructed via modular assembly. Genome Res. 19, 1279-1288.

Li, P., Estrada, J.L., Burlak, C., and Tector, A.J. (2013). Biallelic knockout of the alpha-1,3 galactosyltransferase gene in porcine liver-derived cells using zinc finger nucleases. J. Surg. Res. 181, e39-45.

Mashimo, T., Takizawa, A., Voigt, B., Yoshimi, K., Hiai, H., Kuramoto, T., and Serikawa, T. (2010). Generation of knockout rats with X-linked severe combined immunodeficiency (X-SCID) using zinc-finger nucleases. PLoS One 5, e8870.

McPherron, A.C., Lawler, A.M., and Lee, S.J. (1997). Regulation of skeletal muscle mass in mice by a new TGF-beta superfamily member. Nature 387, 83-90.

Rogers, C.S., Stoltz, D.A., Meyerholz, D.K., Ostedgaard, L.S., Rok hlina, T., Taft, P.J., Rogan, M.P., Pezzulo, A.A., Karp, P.H., Itani, O.A., et al. (2008). Disruption of the CFTR gene produces a model of cystic fibrosis in newborn pigs. Science 321, 18371841.

Schuelke, M., Wagner, K.R., Stolz, L.E., Hubner, C., Riebel, T., Komen, W., Braun, T., Tobin, J.F., and Lee, S.J. (2004). Myostatin mutation associated with gross muscle hypertrophy in a child. N. Engl. J. Med. 350, 2682-2688.

Wiedenheft, B., Sternberg, S.H., and Doudna, J.A. (2012). RNAguided genetic silencing systems in bacteria and archaea. Nature 482, 331-338.

Yang, D., Yang, H., Li, W., Zhao, B., Ouyang, Z., Liu, Z., Zhao, Y., Fan, N., Song, J., Tian, J., et al. (2011). Generation of PPARgamma mono-allelic knockout pigs via zinc-finger nucleases and nuclear transfer cloning. Cell Res. 21, 979-982.

Yu, S., Luo, J., Song, Z., Ding, F., Dai, Y., and Li, N. (2011). Highly efficient modification of beta-lactoglobulin (BLG) gene via zincfinger nucleases in cattle. Cell Res. 21, 1638-1640. 